\title{
The Search for Wolf-Rayet Stars in IC10
}

\author{
Katie Tehrani, Paul Crowther and Isabelle Archer \\ Department of Physics \& Astronomy, University of Sheffield, \\ Hounsfield Road, S3 7RH, Sheffield, UK \\ email: k.tehrani@sheffield.ac.uk
}

\begin{abstract}
We present a deep imaging and spectroscopic survey of the Local Group starburst galaxy IC10 using Gemini North/GMOS to unveil the global Wolf-Rayet population. It has previously been suggested that for IC10 to follow the WC/WN versus metallicity dependence seen in other Local Group galaxies, a large WN population must remain undiscovered. Our search revealed 3 new $\mathrm{WN}$ stars, and 5 candidates awaiting confirmation, providing little evidence to support this claim. We also compute an updated nebular derived metallicity of $\log (\mathrm{O} / \mathrm{H})+12=8.40$ \pm 0.04 for the galaxy using the direct method. Inspection of IC10 WR average line luminosities show these stars are more similar to their LMC, rather than SMC counterparts.
\end{abstract}

Keywords. stars: Wolf-Rayet, galaxies: individual (IC10), ISM: abundances

The Local Group dwarf galaxy IC10, located at a distance of $740 \mathrm{kpc}$, is host to an unusual population of Wolf-Rayet (WR) stars, which are massive stars progressing through the end stages of evolution. Previous WR surveys have identified $26 \mathrm{WR}$ stars, corresponding to an unusually high spectral type ratio of $\mathrm{WC} / \mathrm{WN}=1.3$, in view of the low metallicity environment of IC10 (Crowther et al. 2003). One explanation suggested these prior surveys are incomplete for WN stars (Massey \& Holmes 2002), therefore prompting the deep imaging and spectroscopy presented here.

Using narrow HeII and continuum filters with Gemini North/GMOS, image subtraction techniques were used to reveal HeII excess sources, a robust marker for WR stars. This search yielded $9 \mathrm{WR}$ candidates, and follow up spectroscopy on 4 of these targets successfully confirmed 3 new WN stars. One of these stars, associated with the HII region HL45, provided a clear measurement of the [OIII] $\lambda 4363$ nebular emission line, allowing an updated oxygen abundance to be obtained. Prior metallicity measurements recorded a value of $\log (\mathrm{O} / \mathrm{H})+12 \approx 8.26$ (Garnett 1990), however our analysis suggests a higher value of $\log (\mathrm{O} / \mathrm{H})+12=8.40 \pm 0.04$.

The WR star total in IC10 has now been raised to 29, with an updated WC/WN ratio of 1. With deep imaging revealing a further $5 \mathrm{WR}$ candidates, we are confident a huge hidden population of WR stars does not exist. The sensitivity limit of this survey is $\mathrm{M}_{\mathrm{HeIIC}}=$ -2.4 mag (for $\mathrm{A}_{\mathrm{HeIIC}}=3.4 \mathrm{mag}$ ) which should be sufficient to identify these stars, even those which may fall into the recently proposed new WR class of characteristically faint WR stars (Massey et al. 2014). Average HeII $\lambda 4686$ and CIV $\lambda 5808$ line luminosities for WN and WC stars respectively are comparable to those of LMC WR stars and therefore consistent with the new higher metallicity measurement obtained.

\section{References}

Crowther, P. A., Drissen, L., Abbott, J. B., Royer, P., \& Smartt, S. J. 2003, A\&AA, 404, 483

Garnett, D. R. 1990, ApJ, 363, 142

Massey, P. \& Holmes, S. 2002, ApJ, 580, L35

Massey, P., Neugent, K. F., Morrell, N., \& Hillier, D. J. 2014, ApJ, 788, 83 